\title{
Effect of Duration of Vagal Stimulation on Shortening Velocity of In Vivo Canine Trachealis Muscle
}

\author{
Hiroshi Okayama, Michiko Okayama, Munehiko Ishit, \\ Hidetada Sasaki and Tamotsu Takishima
}

The First Department of Internal Medicine, Tohoku University School of Medicine, Sendai 980

\begin{abstract}
Okayama, H., Okayama, M., Ishit, M., Sasaki, H. and Takishima, T. Effect of Duration of Vagal Stimulation on Shortening Velocity of In Vivo Canine Trachealis Muscle. Tohoku J. Exp. Med., 1989, 158 (1), 37-46 — We studied shortening velocity of in vivo canine trachealis muscle contracted by bilateral vagus nerve stimulation, as a function of duration of contraction. The cervical trachea was transected at two locations, cut at the ventral portion, and opened. One side of the cartilage cut was connected to a force transducer and the other to a lever with a given weight as an afterload, the length of which was measured by a linear displacement transducer. Bilateral vagosympathetic trunks were stimulated by supramaximal electrical impulses. With vagal stimulation, the trachealis muscle started to contract isometrically and at a given time the muscle was allowed to contract isotonically by unlocking a stopper at a given afterload. The shortening velocity was reduced with longer duration of active state. Ten sec after vagal stimulation the maximum force was $730 \pm 105 \mathrm{~g} / \mathrm{cm}^{2}$ (mean \pm S.D.) and maximum velocity at zero load calculated by Hill's equation was $0.092 l_{0} / \mathrm{sec}$. We conclude that the force-velocity relationship of in vivo canine trachealis muscle stimulated by vagus nerves exhibits a time-dependency similar to that in vitro. _- airway mechanics; airway smooth muscle; isotonic contraction; vagal stimulation
\end{abstract}

Constriction of airway smooth muscle controls total and local ventilation by changing airway resistances. During dynamic respiratory maneuvers, the time course of airway caliber is determined by the balance of timing of passively length-cycled airway smooth muscle and shortening velocity of the contracted airway smooth muscle (Sasaki and Hoppin 1979). Stephens et al. (1969) and Antonissen et al. (1979) studied the shortening velocity of airway smooth muscle using excised trachealis muscle strips under electrical field stimulation and found that the force-velocity relationship was hyperbolic and could be described by Hill's equation (Hill 1938). They reported that the shortening velocity of the

Received February 1,1989 ; revision accepted for publication May 6, 1989.

Reprint requests: T. Takishima, M.D., the First Department of Internal Medicine,

Tohoku University School of Medicine, 1-1 Seiryo-machi, Sendai 980, Japan. 
trachealis muscle was slower than that of the striated muscles and suggested that the trachealis muscle could not play a significant role in rapid adjustment of transmural forces transmitted to the airway by the considerably faster striated respiratory muscles. We (Hoppin and Sasaki 1978; Sasaki et al. 1986) and Dillon et al. (1981) studied time-dependent force-velocity relationship of the smooth muscle in vitro and reported that the shortening velocity of smooth muscle was largely dependent on the duration of contraction. We have previously studied the force-velocity relationship of trachealis muscle in situ during vagal stimulation (Okayama et al. 1982) and found that both the force and the velocity exhibited lower values than those stimulated by an electrical field in vitro. Because vagal stimulation is one of the most important mechanisms in regulating airway diameter, we re-examined, in the present study, whether in vivo shortening velocity is time-dependent during vagal stimulation.

Recently, force-length relationships of in vivo trachealis muscle were studied using tracheal rings by Brown et al. (1980). We used a similar technique with tracheal rings as used by Brown et al. (1980) except that we studied isotonic shortening velocity of the trachealis muscle with supramaximal vagal stimulation in anesthetized dogs.

\section{Materials and Methods}

Nine mongrel dogs weighing $14.3 \pm 3.3 \mathrm{~kg}$ were anesthetized with intravenous alpha chloralose $(50 \mathrm{mg} / \mathrm{kg})$ and urethane $(500 \mathrm{mg} / \mathrm{kg})$. Each dog was placed on its back, and the cervical trachea was exposed. The trachea was transected in two places, at about the second ring and the 5th or 7th ring. A longitudinal incision was made along the anterior margin of the trachea. An endotracheal tube was inserted into the caudal part of the trachea and the lungs were ventilated mechanically with a constant-volume ventilator (Aika R-60 Shibata Co., Tokyo).

This middle portion of the cervical trachea, 2.5 to $4 \mathrm{~cm}$ in the length, containing four to six tracheal rings, was used for measurement of the trachealis muscle force velocity relationship. At the cranial and caudal portions of the mid trachea, cartilage and mucous membrane were transected, and care was taken to minimize damage to the posterior aspect of the tracheae, including the pars membranosa, and nerves and vessels which enter the posterior surface of the muscle. The cut ends of the ventral portion of the cartilage were sutured to a light Lucite ${ }^{\circledR}$ hinge with two arms, the ends of which were curved in order to fit to the curvature of the cartilage (Fig. 1). One arm of the hinge was connected to an U-Gage force transducer $( \pm 100 \mathrm{~g}$ DIS $\pm 0.4 \mathrm{mg}$ Type UL, Shinkoh, Tokyo) by a stainless steel rod, and the other arm to a lucite lever, which was connected to a linear displacement transducer (Hewlett Packard 7DCDP, Boston, MA, USA). The lever could be locked by a stopper so that the muscle contracted isometrically or the muscle could be shortened isotonically with a given weight (afterload) attached to the lever. Care was taken to reduce the inertial bounce of the lever by using a damping system with liquid paraffin. Bilateral vagosympathetic trunks were cut and ligatured and distal portions were placed on bipolar silver electrodes for stimulation. The tracheal segment and nerves were embedded in liquid paraffin and were kept at $37^{\circ} \mathrm{C}$ by an electrical heating system. The animal's body was covered with a vinyl bag and the temperature was kept at $37^{\circ} \mathrm{C}$ with a heating pad. End expired $\mathrm{CO}_{2}$ gas was monitored with a capnograph (Godart-Mijnhardt L.V. Type CG-5S 003, Amsterdam, Netherlands). Femoral arterial pressure was monitored with a pressure trans- 


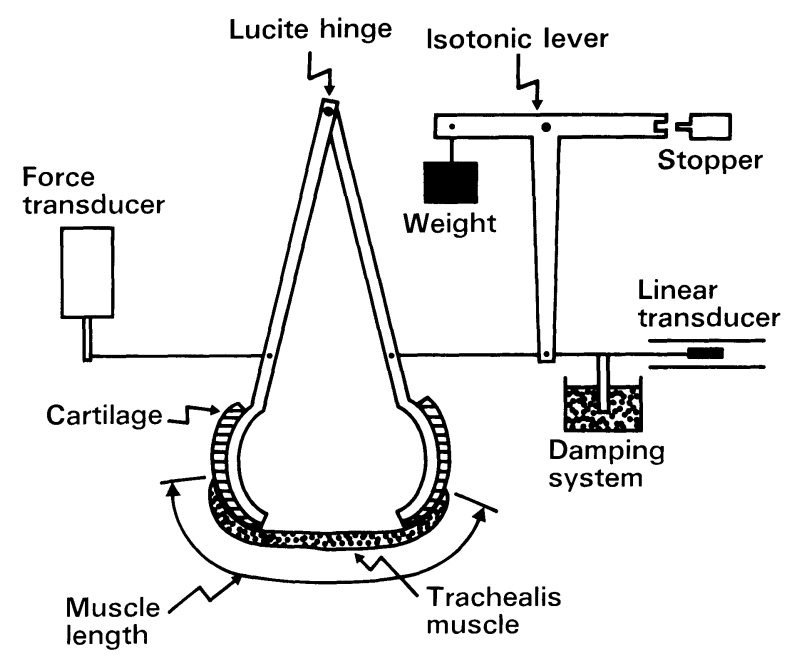

Fig. 1. Schematic diagram of apparatus and preparation. The tracheal cartilage was cut and a hinge with two arms was inserted inside the tracheal cartilage. The arms were curved in order to fit the curvature of the cartilage. Therefore, the trachealis muscle could contract as it does in situ. A force transducer and a linear transducer were connected to the arms.

ducer (MPU-0.5, Nihon Kohden, Tokyo). Arterial gas was frequently sampled and measured with a blood gas analyzer (model 213, Instrumentation Laboratory, Tokyo). The experiments were performed at a systolic arterial pressure of $150 \pm 30 \mathrm{mmHg}$, end expiratory $\mathrm{CO}_{2}$ gas 3 to $4 \%$, arterial $\mathrm{PO}_{2} 78-94 \mathrm{mmHg}$, arterial $\mathrm{PCO}_{2} 34-40 \mathrm{mmHg}$, and $\mathrm{pH}$ 7.29-7.40.

The protocols were as follows. The trachealis muscle was stretched to various lengths and force was measured during supramaximal vagal nerve stimulation at each muscle length. Bilateral vagus nerves were stimulated supramaximally $(3 \mathrm{msec}, 30 \mathrm{~Hz}, 15 \mathrm{~V}$ ) (MSE-3R, Nihon Kohden, Tokyo) for 20-40 sec (Palombini and Coburn 1972). During nerve stimulation heart beat was maintained by artificial right ventricle stimulation (3J06, San-ei, Tokyo). While the stopper on the load lever was locked, isometric contraction was started and the force developed monotonically by vagal stimulation. The force reached a plateau $\left(\mathrm{P}_{0}\right)$ at about 15 sec. Five min were allowed between stimulations in order to avoid fatigue of nerves and muscle (Palombini and Coburn 1972). The muscle length at which the maximum active force was developed was defined as the initial muscle length $\left(1_{0}\right)$ (Brown et al. 1980).

The shortening velocity of the muscle was measured at $1_{0}$ under two conditions.

Effect of duration of contraction on shortening. The dependence of the shortening velocity on duration of contraction was studied in four dogs. After bilateral vagal nerves were stimulated, the muscle contracted isometrically and at a various times after vagal stimulation the stopper was released so that the muscle contracted isotonically with a given load. The velocities were measured at various durations of contraction in random order.

Force velocity study. Using five dogs, force-velocity relationships were measured in the same way, except that the quick release was done $10 \mathrm{sec}$ after vagal stimulation and with various afterloads. When the forces developed during isometric contraction were not reproducible within five percent of the initial force developed or when velocities at a given afterload were not reproducible within $10 \%$, the experiment was discarded. At the end of the experiments, the trachealis muscle was removed, lightly blotted, and weighed in order 
to obtain the force per cross sectional area, which was calculated from the muscle weight, tracheal segmental length and muscle length $\left(1_{0}\right)$ with the assumption of a specific gravity of the muscle of $1.05 \mathrm{~g} / \mathrm{cm}^{3}$ (Stephens et al. 1969). $\quad 1_{0}$ was measured along the curvature of tracheal ring and about $30 \%$ of $1_{0}$ was loosely connected to the tracheal cartilage.

Values were given in terms of mean \pm s.D. Regression coefficient and one-way analysis of variance were used for statistical analysis of data. Significance was accepted at $p<0.05$.

\section{Results}

The mean value and standard deviation of $1_{0}$, the maximum force of the muscle $\left(\mathrm{P}_{0}\right)$, the resting force of the muscle, and the tracheal segmental length of all preparations were $2.7 \pm 0.5 \mathrm{~cm}, 132 \pm 26 \mathrm{~g}, 43 \pm 9 \mathrm{~g}$ and $2.4 \pm 0.4 \mathrm{~cm}$, respectively. (We use the unit of gram weight for force, where $1 \mathrm{~g}$ weight $=980$ dynes.) Fig. 2 shows a typical recording of muscle length and force during isometric contraction followed by isotonic contraction. Ten sec after stimulation, the force was reduced suddenly by removing the stopper and allowing the muscle to shorten at a given force. The change in muscle length can be separated into two distinct phases ; 1. a rapid shortening phase presumably due to recoil of the series elastic component (Stephens and Kromer 1971), and 2. a relatively slow phase of shortening of the contractile elements preceded by transient instabilities. This isotonic quick release method is identical to that used by other workers who have applied the method to skeletal (Jewell and Wilkie 1958), heart (Edman and Nilsson 1968), and smooth muscle (Hellstrand et al. 1972). We calculated the shortening velocity from the linear portion of the slow phase shown by the dashed line in Fig. 2.
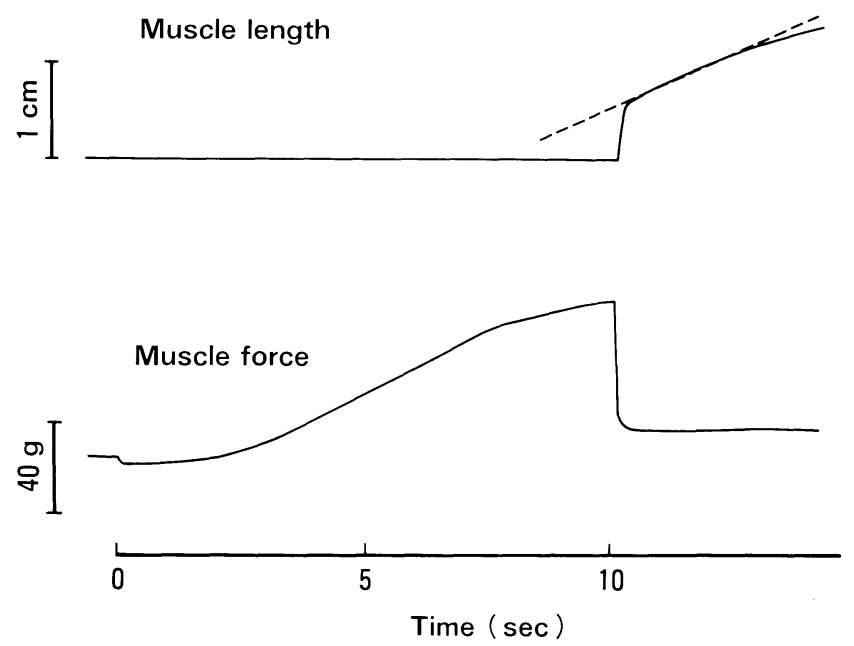

Fig. 2. Response to stimulation of bilateral vago-sympathetic trunks and quick release maneuver. Horizontal scale shows the time. Vagal stimulation was started at time 0 . The muscle became shortening after quick release at an afterload of $55 \mathrm{~g}$. 

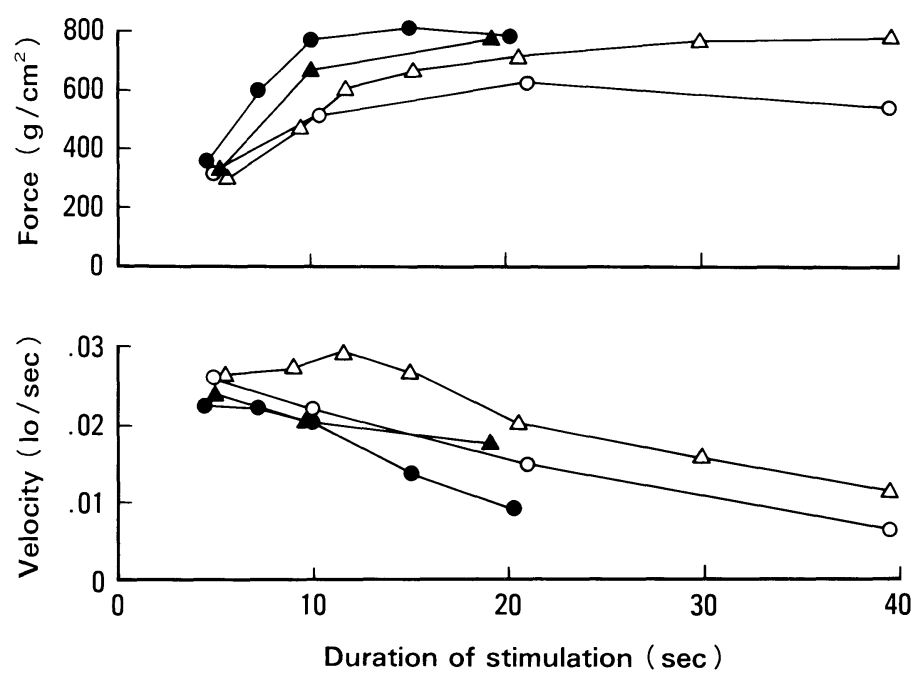

Fig. 3. The upper panel shows muscle force just before the quick release shown in the lower panel. The lower panel shows isotonic shortening velocity with a given afterload (30-40\% of $\mathrm{P}_{0}$ after quick release. Each symbol refers to an individual dog. Afterloads in four dogs shown by $\odot, \triangle, \bullet$ and $\triangle$ are 264, 272, 310 and $327 \mathrm{~g} / \mathrm{cm}^{2}$, respectively.

Fig. 3 (upper panel) shows the developed force just prior to the quick release as a function of duration of contraction. Symbols represent different animals. Fig. 3 (lower panel) shows the shortening velocity just after the quick release, also as a function of the duration of the contraction. Different afterloads were used

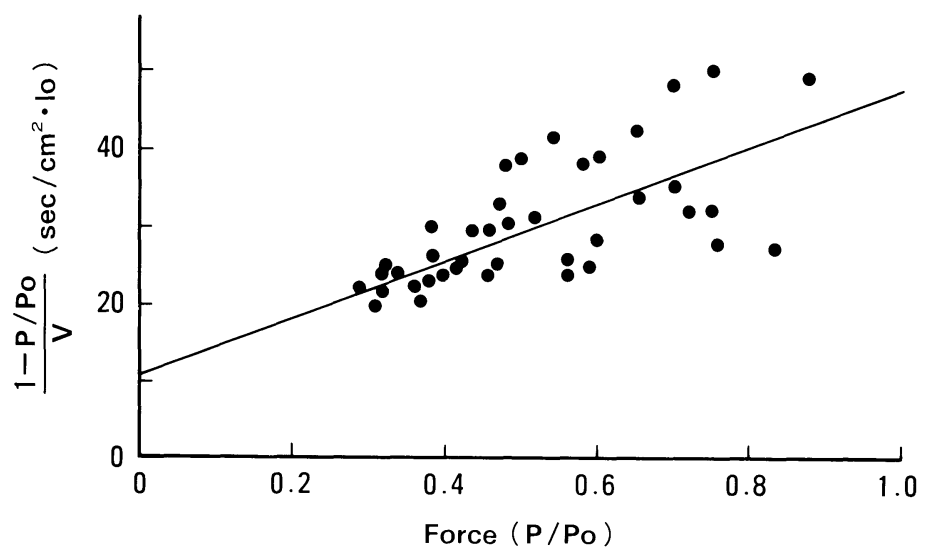

Fig. 4. Force-velocity relations of trachealis muscle obtained from five dogs. The 40 points were gathered by normalizing the force $(\mathrm{P})$ to the maximum force of the muscle of each animal (Peiper et al. 1975). The maximum force and that per square centimeter are $132.1 \pm 26.1 \mathrm{~g}$ and $730 \pm 105 \mathrm{~g} / \mathrm{cm}^{2}$, respectively. 
on different animals. It clearly shows a slower velocity following longer contraction.

Fig. 4 shows the force-velocity relationship obtained from five dogs $10 \mathrm{sec}$ after vagal stimulation. The force $(\mathrm{P})$ was normalized to the maximum force $\left(\mathrm{P}_{0}\right)$ of each animal and plotted on the abscissa, and shortening velocity force $\left(\mathrm{P}_{0}\right)$ of each animal and plotted on the abscissa, and shortening velocity was normalized to initial muscle length $\left(1_{0}\right)$ and shown on the ordinate. Maximum isometric force per square centimeter at the initial muscle length $\left(1_{0}\right)$ of five dogs was $730 \pm$ $105 \mathrm{~g} / \mathrm{cm}^{2}$ (mean \pm s.D.). Since the force-velocity relationship appeared to be a rectangular hyperbola, we tested whether Hill's equation (Hill 1938) could be applied to our data or not. Hill's equation is

$$
(\mathrm{P}+a)(\mathrm{V}+b)=\left(\mathrm{P}_{0}+a\right) \mathrm{b}
$$

where $\mathrm{P}$ represents the force of the muscle, $\mathrm{V}$ the shortening velocity, $a$ a constant with the unit of force, and $b$ a constant with the unit of velocity. Equation (1) may be rearranged as follows.

$$
\frac{1-\frac{\mathrm{P}}{\mathrm{P}_{0}}}{\mathrm{~V}}=\frac{1}{b} \cdot \frac{\mathrm{P}}{\mathrm{P}_{0}}+\frac{1}{b} \cdot \frac{a}{\mathrm{P}_{0}}
$$

In this form, the variable $\left(1-\mathrm{P} / \mathrm{P}_{0}\right) / \mathrm{V}$ is linealy dependent on $\mathrm{P}$. When plotted in this way, we found a linear relationship between $\left(1-\mathrm{P} / \mathrm{P}_{0}\right) / \mathrm{V}$ and $\mathrm{P} / \mathrm{P}_{0}$. The regression equation was $\left(1-\mathrm{P} / \mathrm{P}_{0}\right) / \mathrm{V}=36.7 \mathrm{P} / \mathrm{P}_{0}+10.8(\mathrm{r}=0.70, p<0.01)$ (Fig. $5)$. Therefore, it seems reasonable to assume the force-velocity relationship was hyperbolic and that Hill's equation could be applied. For these experiments $a /$ $\mathrm{P}_{0}$ was equal to 0.293 and $b$ was $0.0271_{0} / \mathrm{sec}$. The shortening velocity at zero

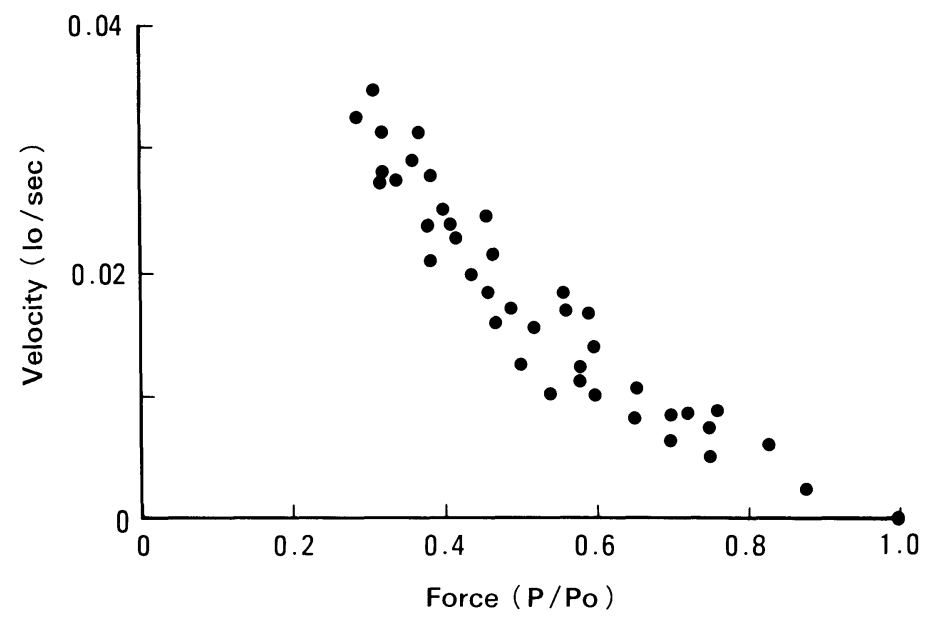

Fig. 5. $\left(1-\mathrm{P} / \mathrm{P}_{0}\right) \mathrm{V}$ are linearly related to $\mathrm{P} / \mathrm{P}_{0}$ : $\left(1-\mathrm{P} / \mathrm{P}_{0}\right) \mathrm{V}=36.7 \mathrm{P} / \mathrm{P}_{0}+10.8(\mathrm{r}=0.70, p<0.01)$. 
load ( Vmax), i.e., the intersection of the curve with the velocity axis, was obtained from the equation (1), as

$$
\mathrm{V} \max =b \mathrm{P}_{0} / a=0.0921_{0} / \mathrm{sec}
$$

When force-velocity curve of the trachealis muscle was plotted in each dog separately, a linear relationship between $\left(1-\mathrm{P} / \mathrm{P}_{0}\right) / \mathrm{V}$ and $\mathrm{P} / \mathrm{P}_{0}$ was also found $(\mathrm{r}=0.68 \pm 0.09$, mean \pm s.D., $p<0.05, n=5)$ and dynamic muscle constants $a, b, \mathrm{a} /$ $\mathrm{P}_{0}$ and $V \max$ were $205 \pm 132 \mathrm{~g} / \mathrm{cm}^{2}, 0.025 \pm 0.0211_{0} / \mathrm{sec}, 0.291 \pm 0.273$ and $0.089 \pm$ $0.0401_{0} / \mathrm{sec}$ (mean \pm s.D.), respectively. Since dynamic muscle constants obtained in each dog separately were not systematically different from those obtained in Figs. 4 and 5, we adopted values calculated from Figs. 4 and 5 in the present study.

\section{Discussion}

Hoppin and Sasaki (1978), Dillon et al. (1981) and Sasaki et al. (1986) reported that with a longer duration of stimulation, the contractile element retained its ability to maintain isometric force but its velocity of shortening was markedly reducecd in excised trachealis or in excised arterial smooth muscle. In the present study we observed that the time dependence of the shortening velocity during supramaximal vagus nerve stimulation of trachealis muscle in vivo behaved similarly to that in vitro. We measured that shortening velocity of contractile elements after $10 \mathrm{sec}$ of vagal stimulation to keep the contraction constant. The shortening velocity at $5 \mathrm{sec}$ was about the same as that at $10 \mathrm{sec}$, despite the fact that $5 \mathrm{sec}$ after vagal stimulation the forces developed were about half those at $10 \mathrm{sec}$ (see Fig. 3). When shortening velocity was observed less than $10 \mathrm{sec}$ after stimulation, developed force is not enough to shorten the muscle with high afterloads. We therefore obtained the force-velocity relationship only at 10 sec. Nonadrenergic inhibitory nerves may affect the time dependent muscle shortening to the vagus nerves. But these nerves are not thought to be functional in dog airways (Suzuki et al. 1976). Therefore, it seems reasonable to assume that the vagi in dogs contain predominantly excitatory nerves.

The trachealis muscle preparation used is the same as that used by Brown et al. (1980), and they measured force-length relationships but not shortening velocity during isotonic contraction. The total maximum force observed by Brown et al. and that of the present study were almost the same. $\mathrm{P}_{0}$ and Vmax reported by Antonissen et al. (1979) were $1211 \mathrm{~g} / \mathrm{cm}^{2}$ and $0.23 \mathrm{l}_{0} / \mathrm{sec}$, respectively. The values of $\mathrm{P}_{0}$ and $V \max$ obtained in the present study are $61 \%$ and $40 \%$, respectively, of the values reported by Antonissen et al. (1979). Muscle in vivo may differ quentitatively from that in vitro, probably due to not only differences in metabolism between in vivo blood perfused muscle and in vitro muscle bathed in physiological salt solution, but also due to differences between vagal and electrical field stimulation. During our experiments both arterial blood gas and 
arterial pressure were maintained within physiological ranges. Thus, the lower velocity of shortening of the trachealis muscle in vivo than in vitro should not have been due to a lower metabolic supply. Under isometric conditions the force of the trachealis muscle after vagal stimulation reached its peak in 10-15 sec (Fig. 3 ), comparable to the values in both time and developed pressures observed in situ studies by Palombini and Coburn (1972). On the other hand, the developed force of the trachealis muscle in vitro under isometric conditions reached its peak in around $8 \mathrm{sec}$ (Stephens and Kromer 1971). The present forces developed by vagal stimulation were lower than those by electrical field stimulations (Stephens et al. 1969 ; Antonissen et al. 1979; Sasaki et al. 1986). The faster rate and higher values of force development under isometric conditions in vitro than in vivo could be due to different method of vagal stimulation resulting in different amount of actomyosin-ATPase activity. With electrical field stimulation a larger amount of transmitter might be released rapidly from the nerve endings. However, during vagal stimulation there might be smaller amounts of release of the transmitter from the nerve endings, resulting in lower rates of muscle force development and slower shortening velocity in vivo than in vitro. The resting force of the present muscle was relatively larger than that reported in in vitro trachealis muscle (Sasaki et al. 1986). Hahn et al. (1976) suggested that with vagi intact there exists basic tone of airway smooth muscle. Since shortening velocity of trachealis muscle exhibits a time-dependency, basic vagal tone may underestimate shortening velocity in the present study.

We have previously estimated the force-velocity relationship of in situ trachealis muscle using an indirect measurement system (Okayama et al. 1982). In that study, we estimated force and velocity by calculation from the volume and the transmural pressure of the closed cervical tracheal segment, based on the assumptions of an ideal model, consisting of a circular cross section for the tracheal tube and a thin and compliant wall for application of Laplace's formula. However, the shape of the cross section was actually not round but depressed at the muscular (membranous) portion in the relaxed state with a smaller tracheal volume reduced by negative pressure. In addition, the effect of the duration of isometric contraction on shortening velocity was not taken into consideration. The present direct measurement method overcomes these problems and makes it possible to obtain more directly the force-velocity relationship of muscle. In the present study, we found that in vivo trachealis muscle $10 \mathrm{sec}$ after vagal stimulation exhibited a hyperbolic force-velocity relationship generally similar to that obtained in in vitro measurement of trachealis muscle (Stephens et al. 1969; Antonissen et al. 1979) and in other smooth muscles (Hellstrand et al. 1972).

Concerning the pathophysiological implications of the change in forcevelocity relationship, Boushey et al. (1980) suggested that it might provide useful information concerning the dynamic properties of bronchial hypersensitivity, since Antonissen et al. (1979) observed an increased shortening velocity 
in vitro from sensitized animals. In the hyperresponsive condition, the process of hyperreaction of the muscle is complex, with contributions not only from the smooth muscle itself but also from neuronal, humoral, and other factors. Further investigation of the force-velocity relationship in vivo as a descriptive tool may be useful in analyzing the dynamics of bronchial hyperreaction. In conclusion, the force-velocity relationship of in vivo canine trachealis muscle stimulated by vagal nerves exhibits mechanical properties with a time-dependence similar to that observed in vitro.

\section{References}

1) Antonissen, L.A., Mitchell, R.W., Kroeger, E.A., Kepron, W., Tse, K.S. \& Stephens, N.L. (1979) Mechanical alterations of airway smooth muscle in a canine asthmatic model. J. Appl. Physiol., 46, 681-687.

2) Boushey, H.A., Holtzman, M.J., Sheller, J.R. \& Nadel., J.A. (1980) Bronchial hyperreactivity. Am. Rev. Respir. Dis., 121, 389-413.

3) Brown, J.K., Leff, A.R., Frey, M.J., Reed, B.R. \& Gold, W.M. (1980) Physiological and pharmacological properties of canine trachealis muscle in vivo. J. Appl. Physiol., 49, 84-94.

4) Dillon, P.F., Aksoy, M.O., Driska, S.P. \& Murphy, R.A. (1981) Myosin phosphorylation and the cross-bridge cycle in arterial smooth muscle. Science, 211, 495-497.

5) Edman, K.A.P. \& Nilsson, E. (1968) The mechanical parameters of myocardial contraction studied at a constant length of the contractile element. Acta Physiol. Scand., 72, 205-219.

6) Hahn, H.L., Graf, P.D. \& Nadel, J.A. (1976) Effect of vagal tone on airway diameters and on lung volume in anesthetized dogs. J. Appl. Physiol., 41, 581-589.

7) Hellstrand, P., Johansson, B. \& Ringberg, A. (1972) Influence of extracellular calcium on isometric force and velocity of shortening in depolarized venous smooth muscle. Acta Physiol. Scand., 84, 528-537.

8) Hill, A.V. (1938) The heart of shortening and the dynamic constants of muscle. Proc. Roy. Soc. Ser. B, 126, 136-195.

9) Hoppin, F.G., Jr. \& Sasaki, H. (1978) Effect of duration of the active state on dynamic properties of airway smooth muscle. Fed. Proc., 37, 553. (abstract)

10) Jewell, B.R. \& Wilkie, D.R. (1958) An analysis of the mechanical components in frog's striated muscle. J. Physiol., 143, 515-540.

11) Okayama, H., Sasaki, H., Ishii, M., Okayama, M. \& Takishima, T. (1982) Isobaric force-velocity relationship of in situ canine trachealis muscle. Respir. Physiol., 48, 323-337.

12) Palombini, B. \& Coburn, R.F. (1972) Control of the compressibility of the canine trachea. Respir. Physiol., 15, 365-383.

13) Peiper, U., Laven, R. \& Ehl, M. (1975) Force velocity relationships in vascular smooth muscle. The influence of temperature. Pflugers Arch., 356, 33-45.

14) Sasaki, H. \& Hoppin, F.G., Jr. (1979) Hysteresis of contracted airway smooth muscle. J. Appl. Physiol., 47, 1251-1262.

15) Sasaki, H., Hoppin, F.G., Jr. \& Takishima, T. (1986) Effect of duration of stimulation on mechanical properties of trachealis muscle. Tohoku J. Exp. Med., 150, 259271.

16) Stephens, N.L. \& Kromer, U. (1971) Series elastic component of tracheal smooth muscle. Am. J. Physiol., 220, 1890-1895.

17) Stephens, N.L., Kroeger, E. \& Metha, J.A. (1969) Force-velocity characteristics of respiratory airway smooth muscle. J. Appl. Physiol., 26, 685-692. 
18) Suzuki, A., Morita, K. \& Kuriyama, A. (1976) Innervation and properties of the smooth muscle of the dog trachea. Jpn. J. Physiol., 26, 303-320. 